\title{
Research on the Transportation Scheme of Urban Emergency Logistics and Materials Based on the Shortest Delivery Time
}

\author{
Xiaoxuan Qi' ${ }^{1,}$, Shize Wang ${ }^{2, b}$,Jingfeng Liür,cand Xiaowei Han ${ }^{1, d}$ \\ ${ }^{1}$ Shenyang University, Shenyang \\ 2 Shenyang yidaxuntong Technology Co.,Itd \\ a13184088@qq.com, b641039302@qq.com, cliu_jingfeng@163.com, dhxw69@163.com
}

\begin{abstract}
Keywords: urban emergency logistics; path optimization; improved genetic algorithm
Abstract. In recent years, the outbreak of natural disasters is becoming more and more frequent, which makes the damage to people's property and life getting worse and worse. Therefore, how to distribute emergency relief supplies after the disaster becomes very important. In this paper, taking the urgent needs of the victims after the disaster into account, based on the shortest delivery time algorithm, a mathematical model of route optimization is established and the model is solved by improved genetic algorithm. The proposed method is applied on a given example with Matlab7.0, and two optimal schemes are obtained, which verified the rationality of the proposed mathematical model and the feasibility of the improved algorithm.
\end{abstract}

\section{Introduction}

With the continuous development of China's economy, a variety of communication equipment gradually come into every household, people are also concerned about the news information. Obviously, all kinds of emergencies often appear in newspapers, television, network, not only in China, Emergencies in many countries in the world are frequent, but China is particularly prominent. The most prominent emergencies of China is natural disasters. China is a country where natural disasters occur very frequently, this is determined by the geographical location of our country. For example, in 2008 the Southern Snowstorm and 5.12 earthquake $^{[1]}$, in 2010 Qinghai Yushu earthquake, on April 20, 2013, Ya'an 7.0 earthquake. According to statistics, nearly 10 years, China's average annual death toll more than 200,000 people caused by various natural disasters, the number of disabled more than 200 million people ${ }^{[2]}$. What amazing figures.

When the disaster occurs, most of the affected areas are without relief supplies reserves, or the reserves of materials are very limited, which can't meet the needs, so the government needs to develop effective rescue measures to ensure that materials can reach the disaster area in the shortest possible time. Efficient rescue operations can effectively control the disaster developments and avoid deterioration of the disaster, greatly reducing the threat of disasters on people's lives and the impact on the national economy ${ }^{[3]}$.

\section{Mathematical Model Based on the Shortest Delivery Time}

Assuming a city has a natural disaster, $m$ areas are subject to different degrees of damage. There are only one emergency materials distribution center around the city, how to plan the distribution route, how to arrange the distribution of vehicles in a reasonable manner, how to transport the medicine, food, tents and other destinations which the victims most needed, all need us to study in depth.

Assumptions: (1)The number of rescue vehicles is less than the number of disaster points, (2) the relief supplies required for each disaster are known and limited, (3) considering the unloading time of the vehicle reaching the affected site and the unloading time per vehicle is known, (4) emergency logistics rescue material transport path optimization problem with hard time window limit (Time Constraint Known), (5) the route between the affected and the affected sites is not completely free, and any roadside collapse may occur at any time.

Symbol Description is as follows ${ }^{[4]}$ :

$m$ : The number of disaster points $i, j(i, j=0,1,2, \ldots, m)$ represents any one of the $m$ disaster points; 
$K$ : The number of rescue vehicles, $k(k=0,1,2, \ldots, K)$ represents any one of the vehicles

$Q:$ The maximum load per vehicle;

$g_{i}:$ The ${ }^{i}$ relief site needed relief supplies and $g_{i} \leq Q, i=1,2, \ldots, m$;

$t_{i j k}$ : The $k$ car from $i$ to $j$ travel time;

The mathematical model based on the shortest delivery time:

$$
\min T=\sum_{i=0}^{m} \sum_{j=0}^{m} \sum_{k=1}^{K}\left(t_{i j k}+t_{i} z_{k i}\right) q_{i j k}
$$

where $\mathrm{T}$ is the total time taken for a vehicle to complete the delivery task for all affected sites ${ }^{[5]}$;

$$
\begin{aligned}
& \sum_{i=1}^{m} g_{i} \cdot y_{k i} \leq Q, \quad \forall k \in K \\
& \sum_{j=0}^{m} q_{k i j}=y_{k i}, \quad i=1,2, \cdots, m, \forall k \in K \\
& \sum_{i=0}^{m} q_{k i j}=y_{k j}, \quad j=1,2, \cdots, \mathrm{m}, \forall k \in K
\end{aligned}
$$

Where (5) is that material requirement for each disaster site is less than the capacity of the disaster relief vehicles; Where(7), (8) are that constraint relations of $y_{k i}$ and $y_{k j}$.

\section{Improved Genetic Algorithm for Optimization of Urban Emergency Logistics Distribution}

(1) Coding and population initialization

The algorithm uses serial number coding, presuming there is only one distribution center 0 , which is the starting point of the vehicle, presuming the number of disaster point is $m$, For the natural number 1 to $m$, there is $K$ of the same rescue vehicles in the distribution center, So there are at most $K$ path for each distribution plan, All rescue vehicles from the distribution center, material distribution is completed and returned to the distribution center, that is, the starting point of the same vehicle. The coding form of each path is made by inserting $K-10$ s between the natural sequences of length $m$ starting at 0 .

Randomly generate the initial population, There are $m$ known distribution center, The initial population size is $n$, When initializing, First ,Set the first position of the chromosome to 0 , The remaining $m$ bits are obtained from a random number of 1 to 3 natural numbers, The randomly generated $n$ chromosome is assigned to each row of the population matrix in turn. Then will produce $n$ Row $m+1$ Column population matrix, The first column of the initial population matrix is 0 , On behalf of the vehicle starting point that distribution center, each row of the matrix represents a chromosome that is a distribution program.

(2) Fitness function

In this paper, the reciprocal of the objective function is used as the fitness function, which is:

$$
F=1 / G=1 /\left(\omega_{1} \cdot G_{1}+\omega_{2} \cdot G_{2}+\omega_{3} \cdot G_{3}\right)
$$

(3) Select operator

The algorithm uses a combination of roulette selection and elite retention options.

(4) Crossover operator

The crossover operator used in this algorithm is an adaptive crossover operator, It $P_{c}$ is not a fixed value, The calculation steps are as follows.

$$
P_{c}=k_{1}\left(f_{\mathrm{m}}-f\right) /\left(f_{\mathrm{m}}-\bar{f}\right)
$$

Among them: 
$f_{m}$ represents the maximum fitness of all individuals remaining in the population of each generation; $\bar{f}$ represents the average fitness value for all individuals remaining in the population with the greatest fitness in each generation; $f$ represents the fitness value of the selected individual; $k_{1}$ represents the formula correction factor, Satisfy $(0,1)$;

Because the father of similar individuals to produce outstanding new individual probability is very low, In order to benefit the evolution of the next generation of traits, In the selection of the parent before crossing, the targeted selection of two individuals with significant differences in traits were crossed. Because with the population in the population similar to the increase in the number of individuals will lead to the ultimate "inbreeding" bad phenomenon, Into the local optimum, So the selection process of the operation is usually guided by the fitness. In order to prevent these shortcomings, we have to take the following measures, Make a threshold before selecting cross parent, If the absolute value of the difference between the fitness values of the two selected parent chromosomes is greater than the predetermined threshold, Otherwise re-select the parent chromosome. In the genetic algorithm, Because the emergence of new individuals is mainly achieved in the cross stage, So individual diversity in the population is realized at this stage.

During the cross operation, After the pairing was completed, the gene was cross-recombined according to the crossover probability $P_{c}$ to produce the offspring chromosome. Using the general cross method, The probability of generating an invalid solution is large, As a result of a repeated crossing of different gene points or After crossing the completion of a chromosome on the gene bit there are multiple 0 together. So choose the two-point cross-improvement method.

(5) Mutation operator

The operator used in this paper is an adaptive mutation operator, The calculation steps of $p_{m}$ are as follows:

$$
P_{m}=k\left(f_{\mathrm{m}}-f\right) /\left(f_{m}-\bar{f}\right)
$$

among them:

$f_{m}$ represents the maximum fitness of all individuals remaining in the population of each generation; $\bar{f}$ represents the average fitness value for all individuals remaining in the population with the greatest fitness in each generation; $f$ represents the fitness value of the selected individual; $k_{2}$ is the formula correction factor, Satisfy $(0,1)$; According to the probability $P_{m}$ of variation.

(6) Termination condition

When the number of iterations reaches a predetermined value of $\mathrm{T}$, the algorithm terminates; otherwise, Number of iterations plus 1.

\section{Experiments and Results}

Re In order to verify the feasibility of the genetic algorithm, we give an example, Suppose a natural disaster occurs somewhere, there are 9 disaster points, with a distribution center and 2 rescue vehicles, distribution centers and disaster point coordinates. The influence factors of the road attributes of the disaster points on the speed of the vehicle. The normal speed of the rescue vehicle is $V=45.5 \mathrm{~km} / \mathrm{h}$, the maximum load is $Q=50$ tons. The discharge time of each disaster point is $0.1 \mathrm{~h}$. 
The algorithm parameters are: the number of iterations is 100 , the population size is 100 , and the formula correction factor $k_{1}, k_{2}$ is 0.5 .

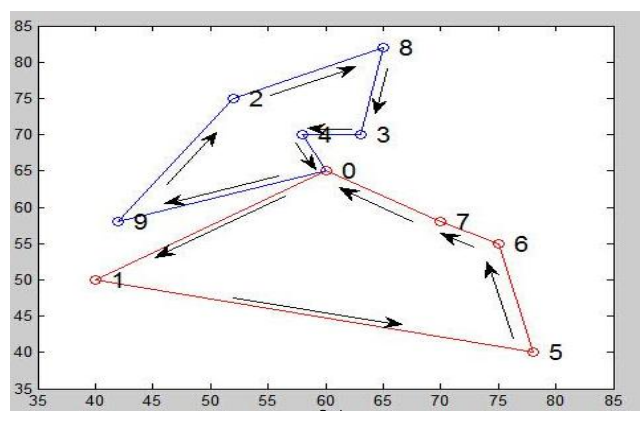

Figure 1 standard genetic algorithm relief supplies distribution routes

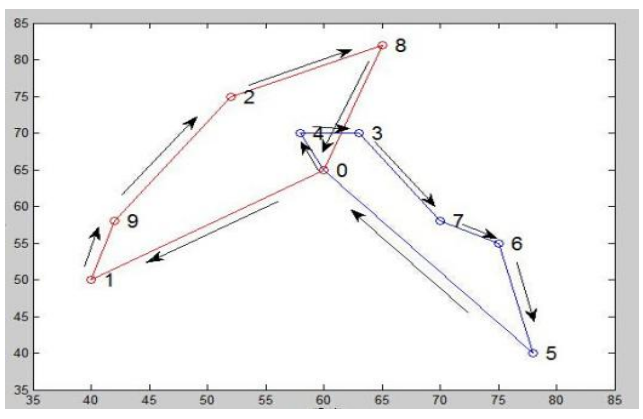

Figure 2 improved genetic algorithm material distribution program

Simulation results with Maltlab 7.0 show the distribution routes that after the disaster, two rescue vehicles transport emergency relief supplies to nine affected areas. .

Distribution of standard genetic algorithms: The distribution route of a rescue vehicle is : $0-9-2-8-3-4-0$, transport delivery time is $3.39 \mathrm{~h}$, The distribution route for another rescue vehicle is: $0-7-6-5-1-0$, transport delivery time is $5.24 \mathrm{~h}$. The total delivery time is equal to the longest vehicle transit time, So the distribution plan with 5.24 hours.

The improved genetic algorithm distribution is: The distribution route of a rescue vehicle is : $0-1-9-2-8-0$, transport delivery time is 4.67 hours, The distribution route for another rescue vehicle is: $0-4-3-7-6-5-0$, the vehicle driving for 4.62 hours.

\section{Conclusions}

Experiments results show that, Improvements of crossover probability, crossover and mutation probability in genetic algorithm can help to increase the diversity of the population, and the experimental results show its effectiveness and efficiency. In the meanwhile, the feasibility of the proposed optimal model is also verified, which paves the way for future research work on transportation of emergency Logistics and Materials under actual conditions. Although the improved genetic algorithm has achieved some results, there are still many works to be further improved.

\section{Acknowledgements}

This work was financially supported by the Liaoning Natural Science Foundation (201602520), and Shenyang Program for Tackling Key Problems in Science and Technology(F15-126-9-00).

\section{References}

[1] Wang W M, Zhao L F, Li J, et al, Rupture process of the M, 8.0 Wenchuan earthquake of Sichuan, China. Chinese J, Geophys.(in Chinese), 2008, 51(5): 1403-1410.

[2] Zhang Y, Xu L S, Chen Y T. Rupture of the Lushan 4.20 earthquake and preliminary analysis on the disaster causing mechanism. Chinese J. Geophys. (in Chinese), 2013,56(4): 1408-1411, doi: $10.6038 / \operatorname{cig} 02130435$.

[3] Zhou C. A method to overcome the convergence of genetic algorithm to local minima [J]. Small Miniature Computer Systems, 2009, 18 (3): 46-49.

[4] Narendra K S. Parthasarathy K. Indentification and Control for Dynamic Systems Using Neural Networks [J].IEEE Trans, Neural Networks, 1990,1(1): 4-27.

[5] Zhang Chunmei, Xing Fei. Using adaptive genetic algorithm to solve the problem of university schedule arrangement [J]. Journal of Inner Mongolia University, 2002,33 (4): $459 \sim 464$. 